\title{
ACRETISMO PLACENTARIO: EVALUACIÓN POR RESONANCIA MAGNÉTICA
}

\author{
JORGE VÉLEZ ${ }^{* *}$, GABRIEL ANGEL ${ }^{2}$, NATHALY BELTRAN ${ }^{3}$, VÍCTOR CALVO ${ }^{4}$ \\ ${ }^{{ }^{*}} \mathrm{MD}$, especialista en Radiología. Investigador de la Fundación Instituto de Alta Tecnología Médica de Antioquia. \\ Medellín, Colombia. \\ ${ }^{2}$ Especialista en enfermería cardiovascular. Jefe de Enfermería e investigador de la Fundación Instituto de Alta \\ Tecnología Médica de Antioquia. Medellín, Colombia. \\ ${ }^{3} \mathrm{MD}$ egresada de la Corporación Universitaria Remington. Medellín, Colombia. \\ ${ }^{4}$ Epidemiólogo e investigador de la Fundación Instituto de Alta Tecnología Médica de Antioquia. Medellín, Colombia. \\ *Correspondencia: Dr. Jorge Mario Vélez, Grupo de Investigación Instituto de Alta Tecnología Médica. Medellín, \\ Colombia.jorgemariovelezarango@gmail.com
}

Dirección postal: Carrera 81 № 30A-99; Medellín. Teléfono: 5203130 ext: 423 - Fax: 211-1717.

Recibido: 26 Mayo 2014 Aceptado: 10 Abril 2015

\section{Resumen}

La placenta acreta es una de las anomalías placentarias más comunes, que incluso puede llevar a la muerte materna y fetal, lo cual se puede evitar con un diagnóstico oportuno.

Objetivo: Describir los hallazgos imaginológicos por resonancia magnética en pacientes con sospecha de adherencia placentaria y correlacionar los resultados con la cirugía.

Materiales y métodos: Estudio descriptivo de series de casos en pacientes con sospecha de invasión placentaria donde se evaluaron los hallazgos de la resonancia magnética frente a los resultados de la cirugía, la cual fue utilizada como estándar de referencia.

Resultados: Se observaron dos casos con acretismo placentario, en los que la resonancia magnética pudo revelar la presencia de lagos venosos anteriores en el primer caso y lagos venosos en el segmento para el segundo caso; ambos con adelgazamiento y discontinuidad de la línea hipointensa que corresponde al miometrio.

Conclusión: La resonancia magnética se convierte en una ayuda diagnóstica útil para establecer la presencia, el grado de infiltración y la penetración de la anomalía placentaria o por el contrario la ausencia de esta patología.

Palabras clave: Acretismo placentario, imagen por resonancia magnética, cesárea, ultrasonografía doppler, edad gestacional. 


\title{
PLACENTA ACCRETA: EVALUATION WITH MAGNETIC RESONANCE
}

\begin{abstract}
Placenta accreta is one of the most common placental abnormalities that can even lead to maternal and fetal death and can be prevented with opportune diagnosis.

Objective: To describe the MRI findings in patients with suspected placental adherence and correlate the results with surgery.

Materials and methods: We present a series of case study in patients with suspected placental invasion to evaluate the findings of MRI compared to the results of the surgery, which was used as the reference standard.

Results: There were two cases of placenta accreta, in which MRI shows previous venous lakes were identified in the first case and venous lakes in the segment for the second case, both thinning and discontinuity of the hypointense line corresponding to the myometrium.

Conclusion: MRI becomes a useful diagnostic tool for establishing the presence, degree of infiltration and penetration of the placental abnormality or conversely the absence of this condition.

Keywords: Placenta accreta, magnetic resonance imaging, cesarean section, ultrasonography doppler, gestational age.
\end{abstract}

\section{PLACENTA ACRETA: AVALIAÇÃO POR RESSONÂNCIA MAGNÉTICA}

\begin{abstract}
Resumo
A placenta acreta é uma das anomalias placentárias mais comuns, que até mesmo pode causar a morte materna e fetal, o que de pode evitar com diagnóstico oportuno.

Objetivo: Descrever as conclusões imaginológicas por ressonância magnética em pacientes com suspeita de adesão placentária e correlacionar os resultados com a cirugía.

Materiais e métodos: Estudo descritivo de series de casos no pacientes com suspeita de invasão placentária donde foram avaliadas as conclusões da ressonância magnética com os resultados da cirurgia, que foi aplicada como padrão de referencia.

Resultados: duos casos com acretismo placentário foram observados, nos que a ressonância magnética poderia revelar a presencia de lagos venosos anteriores no primer caso, e lagos venosos no segmento para o segundo caso; ambos com adelgaçamento y descontinuidade de línea hipointensa que corresponde ao miométrio.

Conclusão: a ressonância magnética troca-se numa ajuda diagnóstica útil para estabelecer a presencia, o grado de infiltração e a penetração da anomalia placentária o pelo contrario, a ausência da patologia que a tratar.
\end{abstract}




\section{Introducción}

La placenta es un órgano de gran complejidad, donde la circulación fetal y materna establecen estrechas relaciones que permiten el intercambio gaseoso y de nutrientes; regula funciones metabólicas y endocrinas que contribuyen al mantenimiento de la gestación y actúa como interface inmunológica entre la madre y el feto (1-6).

En condiciones normales la placenta se adhiere al endométrio y en la mayoría de los casos hacia el fondo uterino. En algunos trastornos de la adhesión placentaria esta puede implantarse por fuera de sus límites normales (acretismo placentario) con cotiledones que se extienden a la pared del útero, estableciéndose diferentes grados: extensión hasta el miometrio sin penetrarlo (placenta acreta), cuando se adhiere al miometrio y lo penetra (placenta increta) y cuando se adhiere, lo penetra y sobrepasa sus límites (placeta percreta) (1-6).

Entre los factores de riesgo para las anomalías placentarias se encuentran: placenta previa, edad avanzada (mayor de 35 años), curetajes, multiparidad, miomectomía, embarazo ectópico, síndrome de Asherman y la presencia de cesáreas previas (1-6), siendo esta última el factor de riesgo más frecuente en el desarrollo de placenta acreta (25\% de los casos) (1-10). De todas las anomalías de la implantación placentaria, la placenta acreta es la más común y la menos severa (7-9).

Las complicaciones placentarias se manifiestan clínicamente con hemorragias en el tercer trimestre del embarazo y con frecuencia, pueden ser tan severas que pueden ser necesarias múltiples transfusiones $(1,2,3,6,7)$; además puede acompañarse de innumerables comorbilidades, entre las cuales se encuentran el parto prematuro, el daño a estructuras adyacentes (vejiga, ureteres, vísceras huecas, estructuras vasculares, entre otros) y en ocasiones en las que se presenta hemorragia severa puede ser necesario el tratamiento urgente con histerectomía como resultado final.

A pesar de los avances en la tecnología médica del siglo XXI se siguen presentando muertes maternas y fetales evitables por estas anomalías, al no contar con un diagnóstico preoperatorio fehaciente; donde en la actualidad la ecografía pélvica y el Doppler son las herramientas diagnósticas más utilizadas $(1,3,5,6)$; dejando como última opción la Resonancia Magnética (RM) debido a su alto costo. La RM ha sido un examen complementario a la ecografía en el diagnóstico de trastornos de la adhesión placentaria cuando los hallazgos son dudosos, no son concluyentes y debido a sus antecedentes se tiene sospecha de trastornos placentarios concomitantes como en el caso del acretismo. La RM posee virtudes sobre la ecografía por el gran campo de visión que nos ofrece y alto contraste de tejidos blandos que permite la visualización de las estructuras placentarias $(1,5)$.

La presente investigación pretende describir los hallazgos imaginológicos por RM en acretismo placentario, la técnica empleada, y a su vez comparar los resultados con la cirugía como prueba de referencia; para ofrecer un diagnóstico oportuno y evitar complicaciones tan severas como la histerectomía e incluso la muerte materna y fetal.

\section{Materiales y Métodos}

Se realizó un estudio descriptivo de serie de casos para caracterizar el acretismo placentario, por medio de la resonancia magnética $(\mathrm{RM})$ en pacientes con o sospecha de patología placentaria; donde se describen las técnicas de la adquisición de la imagen, los diagnósticos clínicos e imagenológicos más representativos en pacientes que fueron referidos a la Fundación Instituto de Alta Tecnología Médica (IATM). De igual manera a las pacientes se les evaluó la edad, la edad gestacional, el número de embarazos y de cesáreas previa.

Descripción de la técnica: Para la adquisición de los exámenes se utilizó un resonador de 1.5 Tesla (Achieva Nova Dual de 16 canales y Achieva de 32 canales, Philips Medical Systems, Best, The Nederlands). Todas las secuencias se realizaron sin medio de contraste y se obtuvieron durante una sola apnea. El protocolo de estudio incluyó el examen desde el diafragma hasta la sínfisis del pubis por medio de imágenes coronales T2 single shot (TR $587 \mathrm{~ms}$ y TE $80 \mathrm{~ms}$ ) de abdomen total, con un FOV (Field of View) de $460 \mathrm{~mm}, 7 \mathrm{~mm}$ de espesor y 20 cortes. Secuencias axiales y sagitales en T2 single shot localizados en la placenta obtenidos durante una apnea con cortes de 3 mm de espesory FOV de 240.

Previa revisión, aprobación y autorización por parte del comité de ética de la Fundación IATM se accedió a la información de los registros clínicos de las pacientes atendidas; los cuales fueron recopilados del RIS (Radiology Information System) y del PACS (Picture Archiving and Communication Systems); de igual manera se solicitaron los hallazgos de la cirugía, a la Institución Prestadora de Servicios que remitió a la paciente.

Esta investigación se clasificó de acuerdo con la declaración internacional de Helsinki, el informe Belmont y la resolución colombiana 8430 de 1993 del Ministerio de Salud como una investigación sin riesgos biológicos, fisiológicos, psicológicos, y sociales. Además se tuvo en cuenta la resolución colombiana 1995 de 1999 por la cual se establecen normas para el manejo de la Historia Clínica. 


\section{Resultados}

Se evaluaron 2 casos de gestantes con sospecha de anormalidad placentaria, donde se describieron algunos aspectos demográficos y de antecedentes clínicos de las gestantes. (Tabla 1.)

Tabla 1. Caracterización demográfica y algunos antecedentes gineco-obstétricos

\begin{tabular}{|c|c|c|c|c|c|}
\hline \#Caso & $\begin{array}{l}\text { Edad } \\
\text { Años }\end{array}$ & $\begin{array}{c}\text { Edad Gestacional } \\
\text { Semanas }\end{array}$ & $\begin{array}{l}\text { \#Gestas } \\
\text { Previas }\end{array}$ & $\begin{array}{l}\text { \#Cesáreas } \\
\text { Previas }\end{array}$ & $\begin{array}{c}\text { Resultado de } \\
\text { Cirugia }\end{array}$ \\
\hline 1 & 36 & 35 & 0 & 0 & Placenta Acreta \\
\hline 2 & 31 & 38 & 7 & 3 & Placenta Acreta \\
\hline
\end{tabular}

\section{Caso 1.}

Paciente de 36 años de edad, primi-gestante, embarazo de 35 semanas, ecografía doppler gestacional con diagnóstico sospechoso de acretismo placentario, lagos venosos, aumento de los vasos placentarios y placenta previa; se ordena resonancia magnética para descartar o confirmar diagnóstico y definir conducta médica. Ver Figura asociado a adelgazamiento en algunas regiones del miometrio que representaron placenta. (Figuras 1, 2 y 3.)
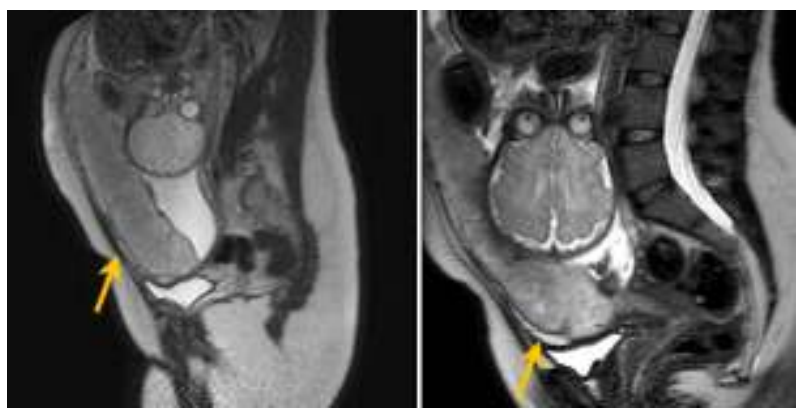

Figura 1. Imágenes sagitales T2, donde se identifica feto único en posición cefálica. Se identificó la presencia de placenta de implantación anterior con múltiples lesiones hiperintensas de comportamiento quístico, consistentes en lagos venosos hacia el segmento uterino anterior e inferior.

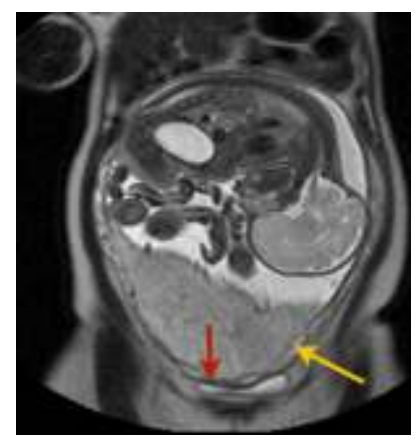

Figura 2. Imagen Coronal T2 del caso 1, donde se identifican los lagos venosos (flecha amarilla) en los segmentos anteriores e inferiores de la placenta, sin aparente extensión de los mismos hacia los órganos vecinos. Se observó la línea hipointensa (flecha roja) correspondiente al miometrio con adecuada visualización de la vejiga.
Figura 3. Secuencia axial T2 donde se vuelve a identificar la presencia de lagos venosos anteriores con adelgazamiento, $a b o m b a m i$ e $n t o y$ discontinuidad de la línea hipointensa que corresponde al miometrio secundario a placenta acreta

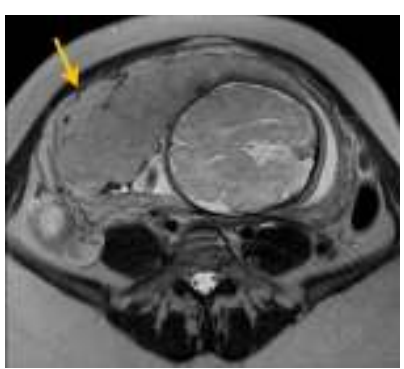

\section{Caso 2.}

Paciente de 31 años de edad, con antecedentes ginecológicos de 3 cesáreas, 4 curetajes, embarazo de 38 semanas, ecografía doppler gestacional con diagnóstico sugestivo de acretismo placentario, se ordena resonancia magnética con énfasis en vejiga y uréter para descartar o confirmar diagnóstico y definir conducta médica. Ver Figura 4,5 y 6 .

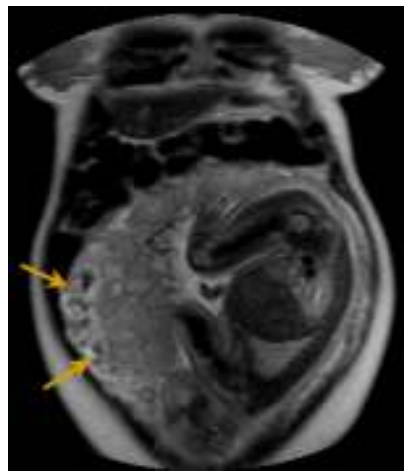

Figura 4. Imagen coronal de abdomen donde se identifican prominentes estructuras vasculares hacia el aspecto antero lateral de la pared miometrial, sin extensión al cuello uterino (flechas amarillas).

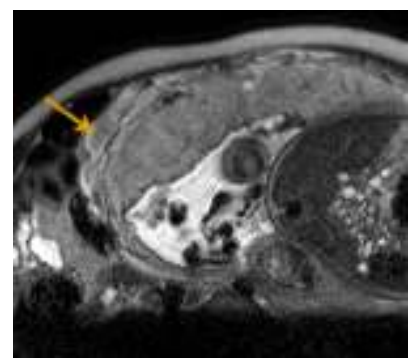

Figura 5. Imagen axial T2 del caso 2, con placenta de implantación anterior donde se identifican lagos venosos $y$ varices prominentes, con mala definición de la línea hipointensa correspondiente al miometrio.

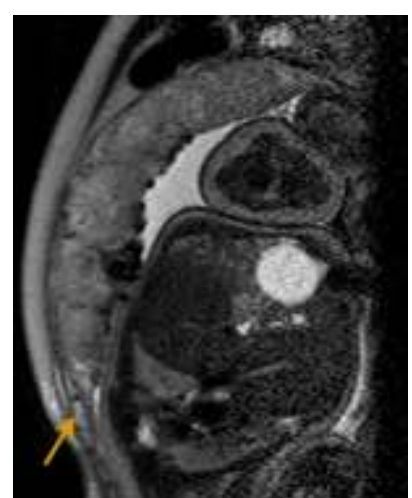

Figura 6. Imagen sagital del caso 2, donde se identifican lagos venosos hacia el segmento, con adelgazamiento de la línea hipointensa que corresponde al miometrio. 


\section{Discusión}

Las alteraciones de la implantación placentaria en la actualidad son un problema que vienen en aumento, debido a múltiples factores de riesgos, como el incremento en el número de cesáreas, la placenta previa, las cirugías uterinas, los legrados uterinos previos, la edad materna mayor de 35 años y la multiparidad (1-12); la placenta previa se asocia con hemorragias potencialmente mortales que pueden ocurrir antes, durante y después del parto, de igual manera es una condición de riesgo sustancial de histerectomía periparto; debido principalmente a la alta frecuencia de placenta acreta (13). (Figura 7)
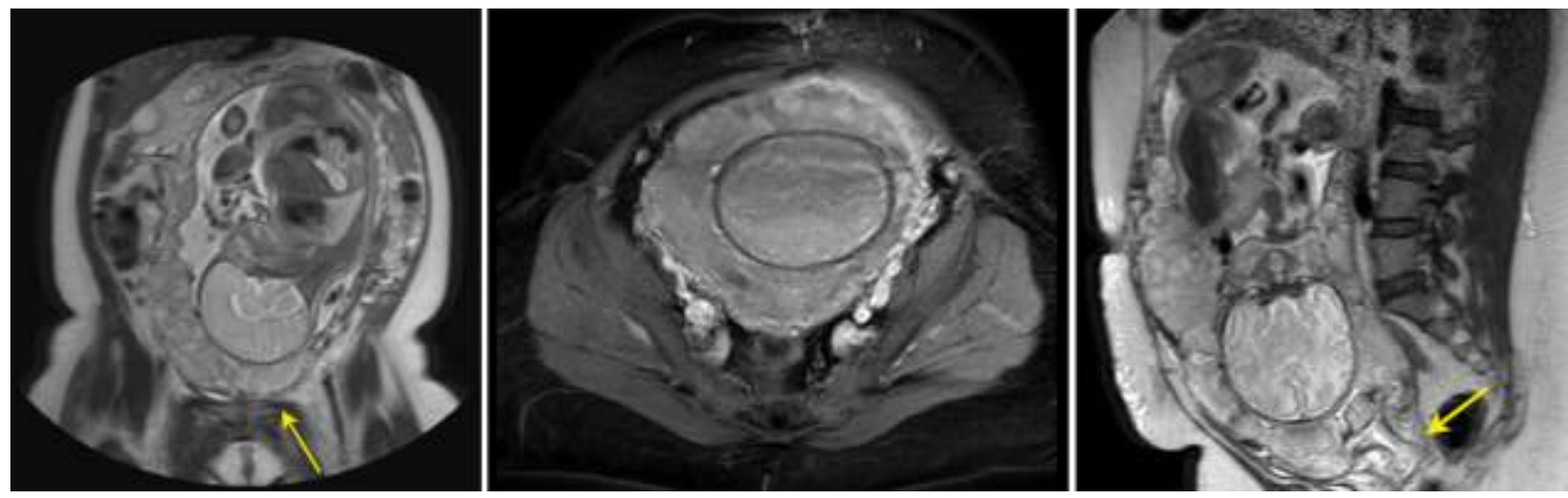

Figura 7. Imágenes Coronal, axial y sagital T2 de abdomen, donde se identifica feto único en posición cefálica con placenta previa total. Nótese los cotiledones placentarios implantados en el tercio inferior del útero, cubriendo el orificio cervical interno por placenta previa total.

Las implantaciones anómalas placentarias son el principal origen de hemorragias obstétricas, siendo esta una de las principales causas de mortalidad materna en el mundo (1416). Con el desarrollo de nuevas tecnologías en ayudas diagnósticas, el profesional médico ha hecho uso de estas herramientas que se encontraban limitadas para ciertas especialidades, permitiendo que la RM tome gran importancia y genere valor agregado al diagnóstico, particularmente en los procesos invasivos de la placenta frente a otras ayudas diagnósticas de menor costo y de mayor accesibilidad como la ecografía doppler; desconociendo adicionalmente que la RM no utiliza radiación ionizante ni medios de contraste yodados, de igual manera proporciona información esencial acerca de patologías subyacentes que pueden conllevar a problemas maternos y fetales; lo cual convierte a la RM en una herramienta útil para el diagnóstico de las adherencias placentarias y las hemorragias intrauterinas, debido a una alta resolución espacial, una buena sensibilidad y una excelente especificidad (88\% y 100\% respectivamente) (17); además la RM alcanza a distinguir la sangre de las colecciones líquidas, del tipo implantación y del grado de infiltración placentaria; permitiendo tomar decisiones frente a la gestión, el manejo y el control de la gravedad de las patologías y sus complicaciones $(15,18)$.

En resonancia magnética, se han desarrollado investigaciones recientes que han permitido generar nuevos protocolos de adquisición de imágenes para resonancia fetal, demostrando que las pacientes que son remitidas a RM y que se encuentran en segundo trimestre de embarazo, pueden tolerar la posición supina durante la obtención de imágenes. En el caso de las pacientes que se encuentran en tercer trimestre de embarazo, se recomienda una posición de cubito lateral izquierdo, que es mejor tolerada por la paciente, y disminuye el riesgo de bajo gasto y compromiso materno, por disminución del retorno venoso (18). Para obtener una adecuada adquisición de las imágenes se recomienda tener a la paciente con la vejiga parcialmente llena, debido a que si esta completamente llena dificulta la visualización de la invasión vesical y afecta el bienestar de la paciente. Tendiendo en cuenta las recomendaciones anteriormente descritas, el especialista logrará observar el adelgazamiento o irregularidad del miometrio subyacente y continuo a la placenta, sin extensión placentaria transmural; extensión placentaria transmural o anormal, intensidad del miometrio confluente con isointensidad de la placenta e invasión de la vejiga por la placenta con irregularidad o disrupción de la arquitectura normal de su pared (19).

Actualmente para el uso de la resonancia de 1.5 Tesla, se recomienda una bobina de cuerpo envolvente o de superficie y para la resonancia de 3 Tesla aun no se ha demostrado que la exposición a altos campos de poder cause algún efecto adverso en el feto $(18,20)$. Masselli y colaboradores (18) recomiendan el uso del siguiente protocolo de resonancia magnética para evaluación de la placenta. (Tabla 2.) 
Tabla 2. Protocolo de RM para la evaluación de placenta tomado de Masselli y colaboradores (18).

\section{Parameter}

Repetition time/echo
time(ms)
Flip angel (ㅇ)

Field of view (mm)

Matrix

Parallel imaging factor

Section thinkness (mm) 4

Intersection gap (mm)

No. Of sections per stack

Time per stack (s)

No. of stack

True fast-imaging
sequence
(true FISP)a

T2 half-Fourier
sequence
(HASTE) $^{\mathrm{b}}$

\section{T1 3D sequence T1 3D
(VIBE)c}

\begin{tabular}{|c|c|c|c|c|c|c|}
\hline Axial & $\begin{array}{l}\text { Corona/ } \\
\text { sagital }\end{array}$ & Axial & $\begin{array}{l}\text { Corona/ } \\
\text { sagital }\end{array}$ & Sagital & Axial & Sagital \\
\hline $4.3 / 2.2$ & $4.3 / 2.2$ & $1000 / 90$ & $1000 / 90$ & $4.1 / 1.1$ & $4.1 / 1.1$ & $\begin{array}{l}3200 / 7 \\
5\end{array}$ \\
\hline 50 & 50 & 150 & 150 & 10 & 10 & 10 \\
\hline $320-400$ & $320-400$ & $320-400$ & $320-400$ & $320-400$ & $320-400$ & $320-40$ \\
\hline $256 \times 224$ & $\begin{array}{l}320 x \\
270\end{array}$ & $\begin{array}{l}256 X \\
224\end{array}$ & $\begin{array}{l}384 \\
X 256\end{array}$ & $\begin{array}{l}256 x \\
256\end{array}$ & $256 \times 224$ & $\begin{array}{l}256 x \\
192\end{array}$ \\
\hline 2 & No/2 & 2 & 2 & 3 & 2 & 2 \\
\hline 4 & 4 & 4 & 4 & 3 & 3 & 5 \\
\hline 0.9-1 & 0 & $0.9-1$ & 0 & 0 & 0 & 0 \\
\hline 35 & $20-24$ & 35 & $20-24$ & 40 & 72 & \\
\hline $20-15$ & $18-10$ & $20-15$ & 16 & $21-18$ & 17 & 180 \\
\hline $2-3$ & 1 & $2-3$ & 1 & 1 & 2 & 1 \\
\hline
\end{tabular}

Diffusion-weighted MR images were acquired with b values of 50,400 , and $800 \mathrm{~s} / \mathrm{mm}$

a The true fast-imaging sequence is the true fast imaging with steady-state free-precession sequence

$\mathrm{b}$ The T2 half-Fourier sequence is the T2-weighted half-Fourier acquisition single-shot turbo spin-echo acquired without and with fat saturation.

Axial Fat saturation was achieved with the chemical shift-selective fat suppression technique

c The T1 three-dimensional (3D) fat-saturated sequence is the T1-weighted dynamic volumetric interpolated breath-hold examination (VIBE) with fat saturation sequence. Fat saturation was achieved with the chemical shift-selective fat suppression technique or frequency-selective adiabatic inversion pulse (SPIRE).
Otras ayudas diagnósticas diferentes de la RM como la ecografía Doppler son más utilizadas para la evaluación y la detección de invasión de la placenta, la cual se realiza en todas las pacientes con sospecha de placenta previa o de acretismo placentario (11). La ecografía es capaz de evaluar de forma óptima la presencia de acretismo, especialmente cuando ocurre en la parte anterior del segmento inferior del útero, siendo esta localización la más común debido a la cicatriz quirúrgica de la cesárea; pero la ecografía no es específica para dar las características de una placenta acreta, increta o percreta, por esta razón las pacientes son referidas a la RM; de igual manera cuando hay sospecha clínica y los resultados de la ecografía no son confiables se hace necesario realizar una RM para evaluar los órganos comprometidos como sucede en la placenta percreta, donde la imagen por RM nos ofrece un gran campo de visión y un alto contraste de tejidos blandos, que permite la visualización de las estructuras placentarias y la afectación de órganos adyacentes $(7,11)$.

Exiten numerosas publicaciones sobre el uso de la RM en el diagnóstico de acretismo placentario, en donde la mayoría de los estudios concuerdan que la RM tiene una exactitud diagnóstica adecuada $(1,3,21)$. Otra de las ventajas que tiene la RM sobre la ecografía Doppler es que cuando la placenta se inserta en la cara posterior del segmento uterino no es posible visualizarse por ecografia, debido a que está queda fuera del campo visual y se dificulta la evaluación de la misma, en estos casos es mejor la información que nos brinda la RM $(13,18)$; por este motivo se recomienda que en las pacientes con alto riesgo de placenta ácreta. Algunos autores refieren que la RM brinda una mejor delineación de planos tisulares, que permiten obtener imágenes anatómicas precisas de la pared uterina, de la vascularización y otras estructuras pelvianas (13). 


\section{Conclusiones:}

El diagnóstico y tratamiento de los trastornos de la adhesión placentaria requieren de un equipo interdisciplinario por tratarse de una patología de difícil diagnóstico y devastadores resultados en el binomio madre hijo. Con el uso más frecuente de la resonancia magnética como complemento al ultrasonido pueden detectarse de forma temprana y oportuna implantaciones anormales de la placenta y la detección de invasión a órganos vecinos intraabdominales. Otras patologías intraabdominales asociadas pueden ser detectadas adicionalmente con el uso de la resonancia magnética y su detección por manos expertas con adecuados parámetros de evaluación.

A pesar del alto costo de la resonancia magnética, su contribución es muy importante en el diagnostico de las patologías placentarias de difícil visualización como las placentas de implantación posterior, o en las que la ecografía Doppler no es concluyente.

\section{Conflicto de intereses}

Los autores declaran no tener de manera directa o indirecta, ningún tipo de conflicto de intereses financieros, académicos o laborales que puedan poner en peligro la validez de este estudio.

\section{Financiación}

Este trabajo se realizó con el apoyo financiero de la Fundación Instituto de Alta Tecnología Médica - IATM. Medellín, Colombia.

\section{Referencias}

1. Masselli G, Brunelli R, Casciani E, Polettini E, Piccioni MG, Anceschi $\mathrm{M}$, et al. Magnetic resonance imaging in the evaluation of placental adhesive disorders: correlation with color Doppler ultrasound. Eur Radiol. 2008;18(6):1292-9.

2. Mazouni C, Palacios-Jaraquemada JM, Deter R, Juhan V, Gamerre M, Bretelle F. Differences in the management of suspected cases of placenta acreta in France and Argentina. Int $J$ Gynaecol Obstet. 2009;107(1):1-3.

3. Palacios-Jaraquemada JM. Diagnosis and management of placenta accreta. Best Pract Res Clin Obstet Gynaecol. 2008;22(6):1133-48.

4. Teo TH, Law YM, Tay KH, Tan BS, Cheah FK. Use of magnetic resonance imaging in evaluation of placental invasion. Clin Radiol. 2009;64(5):511-6

5. Blaicher W, Brugger PC, Mittermayer C, Schwindt J, Deutinger $\mathrm{J}$, Bernaschek $\mathrm{G}$, et al. Magnetic resonance imaging of the normal placenta. Eur J Radiol. 2006;57(2):256-60.

6. El-Bialy G, Kassab A, Armstrong M. Magnetic resonance imagining (MRI) and serial beta-human chorionic gonadotrophin (beta-hCG) follow up for placenta percreta. Arch Gynecol Obstet. 2007;276(4):371-3

7. Bauer ST, Bonanno C. Abnormal Placentation. Semin Perinatol. 2009;33(2):88-96

8. $\quad \mathrm{Ng}$ MK, Jack GS, Bolton DM, Lawrentschuk N. Placenta percreta with urinary tract involvement: the case for a multidisciplinary approach. Urology. 2009;74(4):778-82.

9. Committee on Obstetric Practice. ACOG committee opinion. Placenta accreta. Number 266, January 2002. American College of Obstetricians and Gynecologists. Int J Gynaecol Obstet. 2002;77(1):77-8

10. Dwyer BK, Belogolovkin V, Tran L, Rao A, Carroll I, Barth R, et al. Prenatal diagnosis of placenta accreta: sonography or magnetic resonance imaging?. J Ultrasound Med. 2008;27(9):1275-81.

11. Thia EW, Lee SL, Tan HK, Tan LK. Ultrasonographical features of morbidly-adherent placentas. Singapore Med J. 2007;48(9):799-802.

12. Abehsera DD, Gonzales BC, López MS, Sancha NM, Magdaleno DF. Placenta percreta, experiencia en 20 años del hospital universitario la paz, Madrid, España. Rev chil obstet ginecol. 2011;76(2):127-131.

13. Giambattista E, Ossola MW, Duiella SF, Crovetto F, Acaia B, Somigliana E, et al. Predicting factors for emergency peripartum hysterectomy in women with placenta previa. Arch Gynecol Obstet. 2012;285(4):901-6.

14. Organización mundial de la salud [Internet]: Informe sobre la salud en el mundo 2005 - jcada madre y cada niño contarán!. [acceso 17 julio 2011]. Disponible en: http://www.who.int/ whr/2005/es/index.html

15. Dueñas $\mathrm{O}$, Rico H, Rodríguez M. Actualidad en el diagnóstico y manejo del acretismo placentario. Rev chil obstet ginecol. 2007;72(4): 266-71.

16. Celesia MC, Gómez-Vilches A. Emergencias obstétricas hemorrágicas. Rev. Arg. Anest. 2000;58(6):410-21.

17. Warshak CR, Eskander R, Hull AD, Scioscia AL, Mattrey RF, Benirschke K, et al. Accuracy of ultrasonography and magnetic resonance imaging in the diagnosis of placenta accreta. Obstet Gynecol. 2006;108(3 Pt 1):573-81

18. Masselli G, Gualdi G. MR imaging of the placenta: what a radiologist should know. Abdom Imaging. 2013;38(3):573-87.

19. Grupo Médico Buenos Aires [Internet]: Protocolo para tratamiento y prevencion de las hemorragias obstétricas graves. [acceso 17 Jul 2011]. Disponible en: http:// www.obstetriciacritica.com.ar/doc/difusionprotocolo.

20. Baughman WC, Corteville JE, Shah RR. Placenta accreta: spectrum of US and MR imaging findings. Radiographics. 2008;28(7):1905-16.

21. Lam G, Kuller J, McMahon M. Use of magnetic resonance imaging and ultrasound in the antenatal diagnosis of placenta accreta. J Soc Gynecol Investig. 2002;9(1):37-40. 